\title{
Alternative method's results for the non targeted determination of xenobiotics in food by means of high resolution and accuracy mass spectrometry
}

\author{
Rossana Scarpone* (10), Rachele Rocchi, Federico Bacà, Roberta Rosato, Francesco Chiumiento and \\ Giacomo Migliorati
}

\begin{abstract}
The application of a high resolution and accurate mass spectrometry (HRAMS) approach to detect xenobiotics in different food matrices by means of non targeted determination by UHPLC-Orbitrap followed by data processing analysis was described. Three case studies were reported to demonstrate the possibility to identify unexpected substances in different food commodities overcomes targeted method. This innovative approach could lay the foundation for its applicability to routine analysis in the near future giving the possibility to open new horizons to the research of a wide range of xenobiotics.
\end{abstract}

Keywords: High resolution accurate mass spectrometry, Non-target screening, Food, Xenobiotics, Differential analysis

\section{Introduction}

Nowadays, food safety has become a major issue of public concern and it is a key concern for governments, food industry and manufacturers. Frauds, mistakes and accidents can pollute food with a great deal of chemicals and compromise its safety (Hollender et al. 2019).

For food control, targeted methods are the most applied. The current framework focuses only on substances expected to be found in specific food matrices according to compounds established by authorities in the field. For this reason, these target analyses focus on the detection of one or few classes of compounds. In many cases the extraction procedures are complex and also

\footnotetext{
*Correspondence: r.scarpone@izs.it
}

Department of Bromatology and Residues in Foods for Human and Animal Consumption, Istituto Zooprofilattico Sperimentale dell'Abruzzo e del Molise 'G.Caporale', Via Campo Boario snc, 64100 Teramo, TE, Italy expensive, but enable to lower the analytes detection limits (up to sub ppt-levels) (Kaufmann et al. 2015). But, as a result, new or unexpected substances can go undetected.

Therefore, the development of new strategies is needed in order to ensure a more efficient and rigorous food quality control. The application of a non targeted screening method able to identify a wide range of xenobiotics is an innovative approach involving lower costs and time analyses (Cavanna et al. 2018, Herrera-Lopez et al. 2014). In this approach, target inclusion lists are not used, since the molecules to be detected are not known a priori.

The recent advances in mass spectrometry, mainly high resolution mass spectrometry (HRMS), such as Orbitrap a new type of mass analyzer invented by Makarov (Hu et al. 2005; Makarov and Scigelova 2010; Zubarev and Makarov 2013; Bletsou et al. 2015; Guo et al. 2020; Mol et al. 2012) and Time of Flight (TOF) (García-Reyes et al. 2007; Ki et al. 2019; Peters et al. 2010), together with the use of appropriated software (Milman 2015), original author(s) and the source, provide a link to the Creative Commons licence, and indicate if changes were made. The images or other third party material in this article are included in the article's Creative Commons licence, unless indicated otherwise in a credit line to the material. If material is not included in the article's Creative Commons licence and your intended use is not permitted by statutory regulation or exceeds the permitted use, you will need to obtain permission directly from the copyright holder. To view a copy of this licence, visit http://creativecommons.org/licenses/by/4.0/. The Creative Commons Public Domain Dedication waiver (http://creativeco mmons.org/publicdomain/zero/1.0/) applies to the data made available in this article, unless otherwise stated in a credit line to the data. 
have enabled the development of untargeted approaches (Kaufmann 2014).

These untargeted approaches are a powerful tool for counteracting the continuous development of food contamination with the identification of novel compounds also by using retrospective data analysis. However, at the same time, some critical aspects have also to be taken into account. If, on the one hand, the data collected with this approach need to be evaluated with the use of multivariate statistical models (Riedl et al. 2015), on the other hand a harmonized workflow is required, including standardized protocols and quality requirements. This is to guarantee an efficient framework for data evaluation and communication (Hollender et al. 2019).

Nevertheless, a preliminary attempt of non targeted analysis harmonization was published by the US Pharmacopeia (2016). In this document, the criteria to build a set of reference and test samples able to provide a reliable predictive model are reported. This preliminary work is a good starting point but it is too generic, thus unable to provide suggestions for each analytical technique.

The aim of this work is to apply a high resolution and accurate mass spectrometry (HRAMS) approach to detect xenobiotics in different food commodities by means of non targeted determination by UHPLC-Orbitrap followed by data processing analysis with dedicated compound libraries.

\section{Materials and methods}

\section{Chemicals and reagents}

Acetonitrile $(\mathrm{ACN})$, methanol $(\mathrm{MeOH})$ and water, all LC-MS grade, were purchased from Sigma Aldrich (Steinheim, Germany). Acetic acid (AAc) and formic acid (FAc) for mass spectrometry were also obtained from Sigma Aldrich (Steinheim, Germany). QuEChERS Extraction Packets 5982-7650 (4g of magnesium sulphate, $1 \mathrm{~g}$ of sodium citrate, $0.5 \mathrm{~g}$ sodium hydrogen citrate sesquihydrate, $1 \mathrm{~g}$ of sodium chloride) were obtained from Agilent Technologies (Santa Clara, USA). Finally, Pierce LTQ Velos ESI Negative and Positive Ion Calibration Solution were provided by Thermo Fisher Scientific (Waltham, MA, USA).

In the acquisition, lock masses were used: in positive ionization (ESI+) was disooctyl phthalate $[\mathrm{M}+\mathrm{H}]^{+}$with $\mathrm{m} / \mathrm{z}=391.28429$ and in negative ionization (ESI-) was formic acid dimer $\left[\mathrm{M}_{2}+\mathrm{Na}-2 \mathrm{H}\right]^{-}$with $\mathrm{m} / \mathrm{z}=112.98563$.

\section{UHPLC-HRAMS analysis}

The analysis was based on a previous work from the same laboratory as described in 2020 by Scarpone et al. Briefly, the UHPLC chromatographic analysis was performed using Dionex ${ }^{\mathrm{TM}}$ Ultimate 3000 (Thermo Scientific ${ }^{\mathrm{TM}}$, San Jose, USA) equipped with an analytical column Thermo
Scientific ${ }^{\mathrm{TM}}$ Accucore $^{\mathrm{TM}}$ aQ $\quad$ C18 column $(100 \times 2.1 \mathrm{~mm}$ with particle diameter of $2.6 \mu \mathrm{m}$ ). The oven and autosampler temperature were set, respectively, at $40^{\circ} \mathrm{C}$ and $15^{\circ} \mathrm{C}$. The injection volume was $10 \mu \mathrm{L}$. The mobile phases consisted of water (A) and methanol (B) both containing $5 \mathrm{~mm}$ ammonium formate and $0.1 \%$ of FAc. The analysis was done at a flow rate of $0.4 \mathrm{~mL} \mathrm{~min}^{-1}$ using the following gradient elution: at the beginning, $20 \%$ phase B was constant for $0.5 \mathrm{~min}$, and it was increased up to $98 \%$ in $10 \mathrm{~min}$. The latter was maintained for $4 \mathrm{~min}$, and then switched back to the initial $20 \%$ in $0.5 \mathrm{~min}$ and kept constant for $4 \mathrm{~min}$ giving a total runtime of $19 \mathrm{~min}$. The UHPLC system was connected to the single stage Orbitrap mass spectrometer Q Exactive ${ }^{\mathrm{TM}}$ from ThermoFisher Scientific (Bremen, Germany) through a heated electrospray interface (HESI-II) operating in positive/negative ionization (Makarov and Scigelova 2010). The HESI parameters in positive polarity were the following: electrospray voltage of $3.2 \mathrm{kV}$; sheath gas of 40 arbitrary units; and auxiliary gas of 25 arbitrary units; capillary temperature $250^{\circ} \mathrm{C}$ and auxiliary temperature $300^{\circ} \mathrm{C}$. Instead, in negative polarity were: electrospray voltage of $3.8 \mathrm{kV}$; sheath gas of 40 arbitrary units; and auxiliary gas of 15 arbitrary units; capillary temperature $250^{\circ} \mathrm{C}$ and auxiliary temperature $250^{\circ} \mathrm{C}$.

The analysis was performed with 4 consecutive UHPLC-HRAMS runs in ESI+: 3 with a FullScan-All Ion Fragmentation (FullScan-AIF); 1 in Data Dependent Scan Mode (ddMS2).

The FullScan-AIF runs were acquired with resolving power of 140,000 FWHM for parental ions and mass range of $110-1200 \mathrm{~m} / \mathrm{z}$, AGC target of 3e6, max IT $500 \mathrm{~ms}$; the FullScan-AIF acquisition of all fragments was set with a resolving power of 35,000 FWHM and $63.3-700 \mathrm{~m} / \mathrm{z}$ as mass range, AGC target of $3 \mathrm{e} 6$, max IT $150 \mathrm{~ms}$. The data dependent scan mode run was set with a "Homemade Exclusion list" of about 70 compounds in a mass range from 110 to $950 \mathrm{~m} / \mathrm{z}$ and carried out with resolving power of 70,000 FWHM for parental ions and 17,500 FWHM for all fragmentation products, using a mass accuracy $\leq 2 \mathrm{ppm}$. All the ions that are present in the "environmental-laboratory system" were included in an exclusion list that was generated by performing the blank reagent chromatographic run. All the chromatographic runs were carried out using a stepped energy collision of 20,35 , and $60 \mathrm{eV}$.

Other 4 consecutive UHPLC-HRAMS runs in ESIwere carried out: 3 with a FullScan-All Ion Fragmentation (FullScan-AIF); 1 in Data Dependent Scan Mode (ddMS2). The resolving power, mass range and stepped energy collision were the same set for the positive acquisition mode. Instead, the "Homemade Exclusion list" was not used. 


\section{Case histories and analytical procedure}

The technological improvements in mass spectrometry give new possibilities for greatly increasing the scope of multi-residue methods (MRM) analysis helping the everyday work carried out by laboratories. Whereas full-scan measurements are theoretically the best approach for MS screening, developments in targeted measurements also offer the potential for a substantially increased scope of analysis.

The reason to conducing proficiency tests on screening methods was to gather information from laboratories as to the type of software they use for processing data and to evaluate the analytical method applicability in routine analysis.

The collected data from the participants, used by EU Reference Laboratories for Residues of Pesticides, could help the improvement of data processing software.

In these analyses, for the qualitative screening methods, the acceptable false-negative rate was $5 \%$ according to SANTE/12682/2019 Document and to the 2002/657/ CE Commission Decision (Commission Decision n. 657/ EC 2002, European Commission Directorate General for Health and Food Safety, 2019).

\section{Case study 1}

Our laboratory was involved in a proficiency test (RIKILT test 2018-11), organized by Wageningen University \& Research, for unknown anti-microbiologically active compounds in water in order to test and validate procedures for identification of 'unknown' substances having antimicrobial activity. Our laboratory was identified by lab code number 9837.

Two delivered samples (sample material 1 and 2) consisted approximately of $20 \mathrm{~mL}$ of rain water. The participating laboratories were asked to identify all microbially active compounds in the samples and to report only the identity of these. Quantification was outside of the scope of this test.

The water samples were analyzed without any extraction, concentration and/or clean-up. The analysis has been performed in positive and negative ionization mode, in presence and absence of salt in the mobile phases of UHPLC analysis. The blank matrix used to mapping the matrix components was the Milli-Q water.

\section{Case study 2}

The second case study reported the European Union Reference Laboratory-Fruit and Vegetable-10 Proficiency Test (EURL-FV10) in which the sample matrix was green beans with pods. The lab code of our laboratory was 067 . This Proficiency Test was organized by the EURL for
Pesticide Residues in Fruits and Vegetables and regarded the untargeted screening of pesticides using multi-residue methods analysis. The samples were spiked with 17 pesticides, but the participants did not received information about the list.

The blank matrix was characterized by the same beans grown in the same field.

Two hundred grams portions of homogenate samples were weighed out into screw-capped polyethylene plastic bottles, sealed and stored at $-20^{\circ} \mathrm{C}$ prior the distribution to the participants. The extraction was carried out as reported by Scarpone et al. (2020) with the use of QuEChERS (EN 15662:2008 2008) without clean-up. The sample extracts were diluted 1:10 in water and injected in LC-MS for the analysis.

It was asked to participant laboratories to screen the test items using a wide-scope screening methods normally applied for official monitoring purposes. The evaluation test was based on qualitative information, but it was also requested to estimate the concentration, only for informative purposes.

\section{Case study 3}

The third case study consisted in the EURL-FV-12 Proficiency Test. It was based on the pesticide-residue analysis of onion. The lab code of our laboratory was 053 . This Proficiency Test, like the EURL-FV-10, was also organized by the EURL for Pesticide Residues in Fruits and Vegetables and regarded the untargeted screening of pesticides using multi-residue methods analysis.

Approximately $200 \mathrm{~g}$ of onion test item treated with pesticides were stored at $-20^{\circ} \mathrm{C}$ prior to shipment to participants. The blank matrix sample was not the same of the delivered one. The selected pesticides used to spike the sample were 17 and no information was available to the laboratories.

Also in this case, the extraction was carried out as reported by Scarpone et al. (2020) with the use of QuEChers (EN 15662:2008 2008) without clean-up. The sample extracts were diluted 1:10 in water for the LC-MS analysis.

The aim of this test was to evaluate laboratory capability using large-scope quantitative and screening methods during routine analysis, for detecting and identifying unexpected pesticides at levels at, or above $0.01 \mathrm{mg} / \mathrm{kg}$.

\section{Database searching}

For this work different software and databases were used to identify the unknown compounds, such as online libraries (ACToR: Aggregated Computational Toxicology Resource, Drug Bank, FDA, Nature Chemical Biology, Nature Communications, PubMed, Nature Chemistry, Royal Society of Chemistry, Science Base, Springer 
Nature), Compound Discoverer 2.0, and also the library from Thermo Fisher (EFS HRAM Compound Database).

\section{Results and discussion Method applicability and advantages}

The proposal method allows the identification in a single sample of a wide range of xenobiotics, not previously preselected, that can be detected by means of untargeted screening methods accomplished by UHPLC-Orbitrap followed by data processing analysis. This method could be applied to a variety of food matrices and raw data can be processed many times for performing chemometric and differential analyses.

The analyses of different matrices could present some problems due to the different matrix effect, that consists in an ion suppression or enhancement of analyte caused by co-eluting matrix components (Uclés et al. 2017). This problem can be reduced with the dilution of the extract in most of the cases as also reported by Ferrer et al. (Ferrer et al. 2011). The dilution has the advantage to reduce the matrix effect but at the same time to lower the LODs of the analytes that risk not being detected. The occurring phenomena of signal suppression or enhancement in MS detection cannot be attributed to only one cause, but it depends on a synergic effect of all the conditions involved. The interfering compounds, that co-elute in the chromatographic separation, can be components of the sample, or released during the extraction process or reagents added to the mobile phase (Furey et al. 2013; Gosetti et al. 2010).

\section{Case study 1}

Any information was available on the chemical and physical characteristic of the unknown compounds and to prevent their stability during the analytical procedure and to avoid their loss, no treatment was applied to the samples. The focus was to improve the sensitivity of the screening method and also improve the data evaluation procedures.

The information collected from HRAMS allows the identification of unknown compounds with relatively high degree of confidence without reference analytical standards. The identification was carried out only using the mass spectra database. The test analyzes were carried out directly on the two samples of water delivered for the proficiency test using two analytical columns. These selected columns have similar characteristic; they have the same dimensions $(100 \times 2,1 \mathrm{~mm})$ and both were packed with spherical solid core particles. The only difference was the particle size dimension $(1.8$ and $2.6 \mu \mathrm{m})$. They were tested considering their optimal retention of a wide range of polar analytes with good speed and resolution thanks to their solid-core particles. The first tests were carried out with Thermo Scientific $^{\mathrm{TM}}$ Accucore $^{\mathrm{TM}}{ }^{\mathrm{a}} \mathrm{Q}$ C18 column in positive and negative modes, in presence and absence of salts in the mobile phase of the UHPLC analysis. Then, Acquity C18 column was also tested in the same conditions of the first one. Analyzing the chromatograms, it was observed that several signals presented a better separation, peak shape and efficiency using the Accucore ${ }^{\mathrm{TM}} \mathrm{aQ}$ C18 column in presence of salts in the mobile phases. In all trials in absence of salts, no significant signals were detected, and any compounds were identified.

After these trials, a differential analysis between the blank matrix and the samples was carried out. The blank matrix was not the same of the samples, but as reported in a previous study (Scarpone et al. 2020), it is not significant to use the same blank matrix.

To identify the compounds, different libraries were consulted (see paragraph 2.4).

For the sample material 1, the results of the different trials gave a double putative identification matching to the library: linezolid $\left(\mathrm{C}_{16} \mathrm{H}_{20} \mathrm{FN}_{3} \mathrm{O}_{4}\right)$ and vepandavir $\left(\mathrm{C}_{13} \mathrm{H}_{32} \mathrm{~N}_{6} \mathrm{O}_{3} \mathrm{P}_{2}\right)$. Easily, it was possible to identify putatively in positive mode the first compound as correct based on accurate mass measurements and informative fragmentation spectra matching with the library database (Supplementary Information, Additional file 1a).

For the sample material 2, analysing the results in FullScan-AIF range $[110,0000-1200,0000 \mathrm{~m} / \mathrm{z}]$, it can be claimed that the differential analysis performed by Compound Discoverer 2.0 allowed the putative identification of boromycin, boromycin deprotonated and also $\mathrm{N}$-formyl-boromycin (boromycin-CHO) both in positive and negative mode and in presence of salts.

In Additional file 1b (Supplementary Information), the putative identification of boromycin $\left(\mathrm{C}_{45} \mathrm{H}_{74} \mathrm{BNO}_{15}\right)$, has been shown $\left([\mathrm{M}+\mathrm{H}]^{+}=880.52014 \mathrm{~m} / \mathrm{z}\right)$ at $10.98 \mathrm{~min}$.

Then, the analysis was performed in Data Dependent Scan (ddMS2) to confirm the previous identification. Operating in ESI- mode, the boromycin was detected at $878.52069 \mathrm{~m} / \mathrm{z}$ (Fig. 1a) with a retention time of $11.05 \mathrm{~min}$ with a specific spectra.

The boromycin profile pattern was characterized by $879.52356 \mathrm{~m} / \mathrm{z} \quad\left(\mathrm{C}_{45} \mathrm{H}_{74} \mathrm{BNO}_{15}{ }^{+}\right) ; \quad 878.52069 \mathrm{~m} / \mathrm{z}$ $\left(\mathrm{C}_{45} \mathrm{H}_{73} \mathrm{BNO}_{15}{ }^{+}\right) ; \quad 877.52393 \mathrm{~m} / \mathrm{z} \quad\left(\mathrm{C}_{45} \mathrm{H}_{72} \mathrm{BNO}_{15}{ }^{+}\right)$and $880.52612 \mathrm{~m} / \mathrm{z}\left(\mathrm{C}_{45} \mathrm{H}_{74} \mathrm{BNO}_{15^{-}} \mathrm{H}^{+}\right)$.

The characteristic fragments that contributed to the putative identification were $752.42841 \mathrm{~m} / \mathrm{z}$, $666.58533 \mathrm{~m} / \mathrm{z}, \quad 155.10989 \mathrm{~m} / \mathrm{z}$ and $\quad 116.07271 \mathrm{~m} / \mathrm{z}$ (Fig. 1b).

In ESI+ mode, the same compound was detected as $[\mathrm{M}+\mathrm{H}]^{+}$with a $\mathrm{m} / \mathrm{z}=880.51776$ at $\mathrm{RT} 10.96 \mathrm{~min}$ 


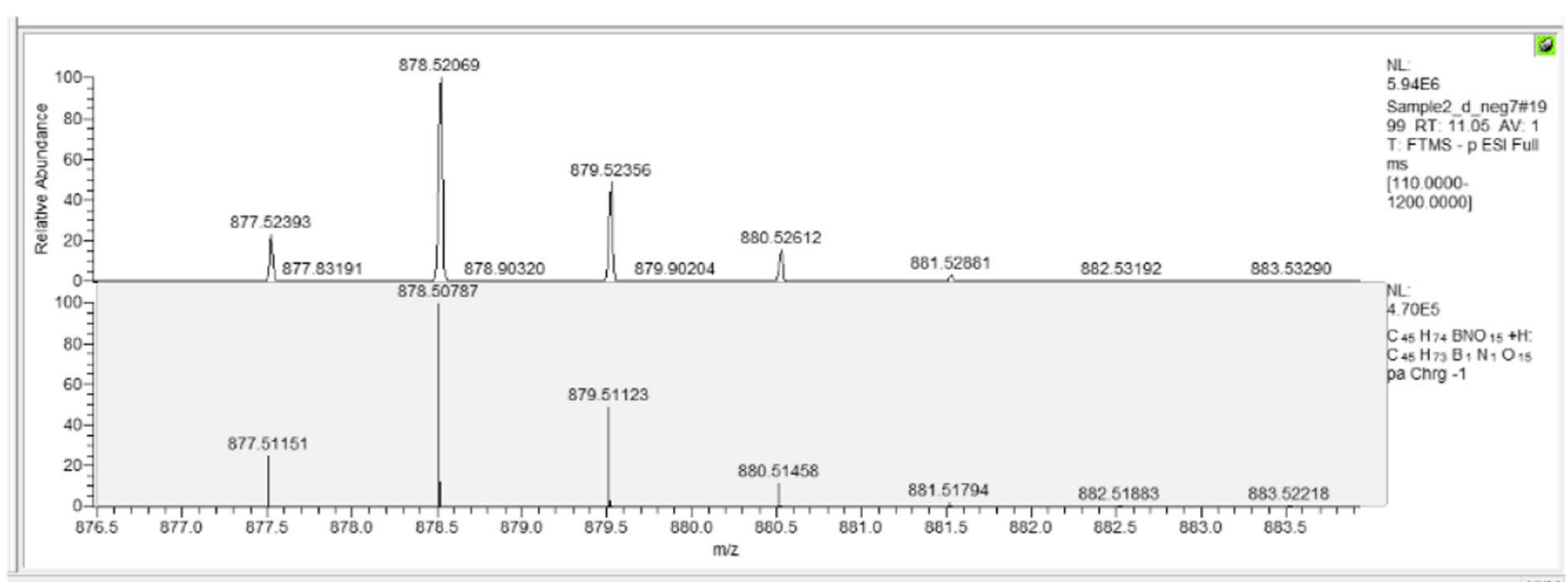

(a)

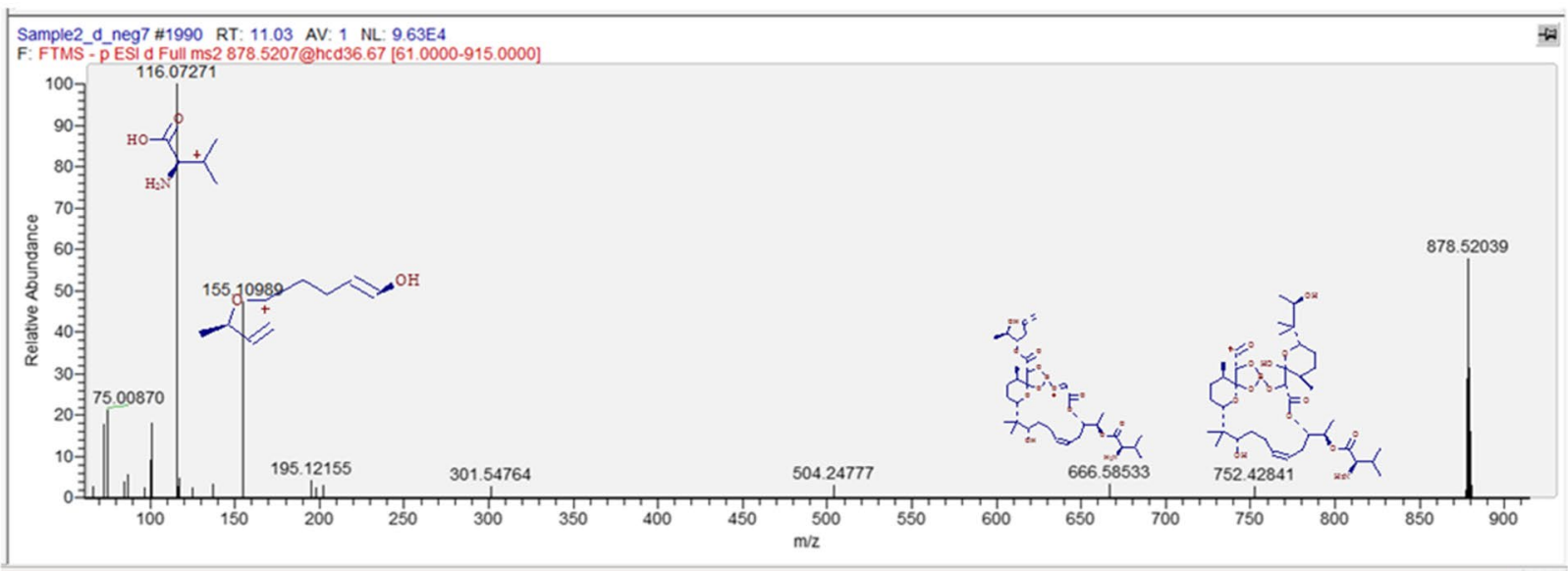

(b)

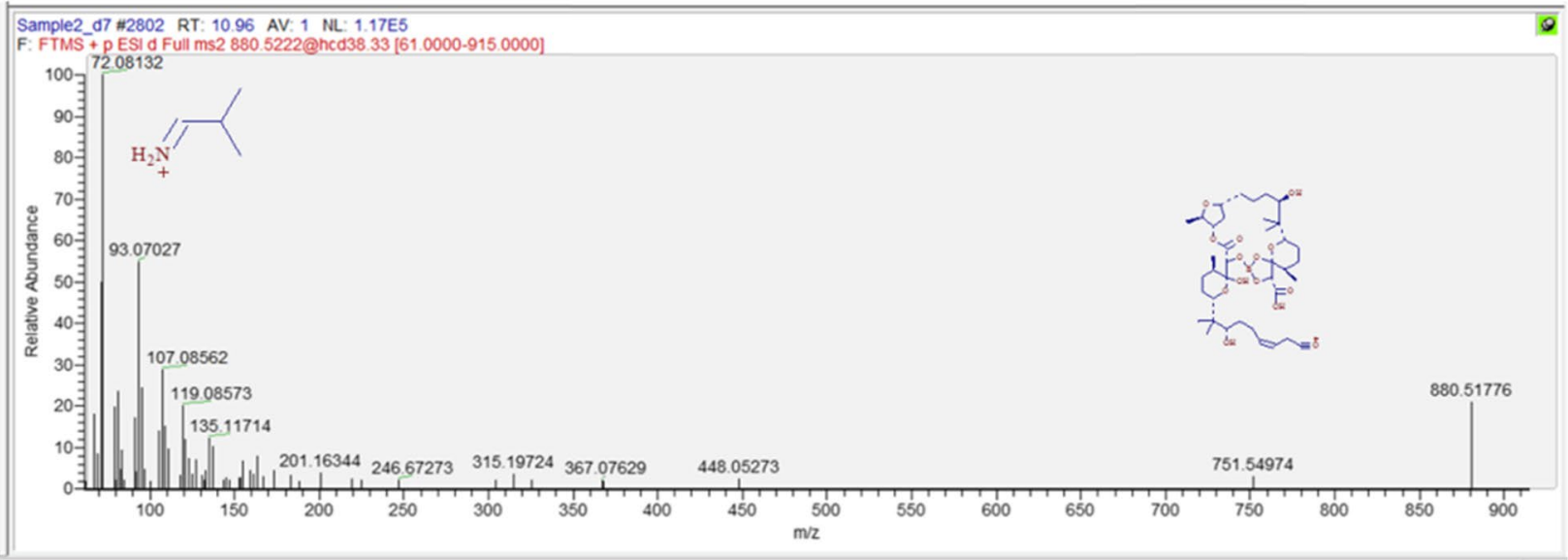

(c)

Fig. 1 Putative identification of Boromycin $\left(\mathrm{C}_{45} \mathrm{H}_{74} \mathrm{BNO}_{15}\right)$ : a Full-Scan in ESI-: experimental and theorical spectra; b ddMS2 acquisition in ESI-: parental ion and fragmentation products $\mathbf{c}$ ddMS2 acquisition in ESI+: parental ion and fragmentation products 
and the fragmentation pattern was characterized by two fragments 751.54974 and $93.07027 \mathrm{~m} / \mathrm{z}$ (Fig. 1c). It was detected also the boromycin adduct with $\mathrm{Na}$ at $902.50049 \mathrm{~m} / \mathrm{z}$ (Supplementary Information, Additional file 1b).

Furthermore, the theoretical fragmentation pattern of boromycin was also studied to confirm the putative identification and to avoid to bump into isobaric compounds.

\section{Case study 2}

The results of this Proficiency Test were reported in the EUPT-FV-SM10 Final Report published by EURL. The pesticides used to spike the samples, at different levels between $0.01 \mathrm{mg} / \mathrm{kg}$ and $0.1 \mathrm{mg} / \mathrm{kg}$, were decided upon the Quality Control Group. The pesticide treatments were carried out just post-harvest used pesticide analytical standards. The selected pesticides were: benalaxyl, clomazone, cyfluthrin, emamectin, etoxazole, fenpyrazamine, isopyrazam, metrafenone, penflufen, pentachloroaniline, penthiopyrad, proquinazid, prosulfocarb, spinetoram, spirotetramat, spirotetramat metabolite BYI08330-enol, sulfoxaflor and tetramethrin. Seventy-three laboratories participated to this Proficiency Test and only 69 of them submitted the results. These laboratories analysed the test item using method based on gas or liquid chromatography, or both, combined with mass spectrometry detection.

Fourteen pesticides, corresponding to $78 \%$ of reported pesticides by laboratory, were detected in our laboratory by liquid chromatography analysis combined with high resolution mass spectrometry.

It was possible in our laboratory to detect and identify 14 pesticides:

benalaxyl, clomazone, emamectin, etoxazole, fenpyrazamine, isopyrazam, metrafenone, penflufen, penthiopyrad, prosulfocarb, spinetoram, spirotetramat, sulfoxaflor and tetramethrin. Three pesticides were not detected: cyfluthrin, pentachloroaniline and proquinazid. In particular, the detection of cyfluthrin and pentachloroaniline was failure because they are analytes suitable for the GC-MS determination.

Instead, proquinazid was not detected with our screening method because its signal was low, and it was not depart from the blank matrix with the set statistical parameters ( $p$-value $<0.05$ and log fold charge $=1$ ). The differential analysis showed about 600 compounds to be identified, but the proquinazid signal was not included in this group, but present among the matrix signals. In retrospective analysis, it was present a low signal of the parent ion at $[\mathrm{M}+\mathrm{H}]^{+}=373.04000 \mathrm{~m} / \mathrm{z}$ and also its ion fragments at 330.99480 and $288.94750 \mathrm{~m} / \mathrm{z}$, recognized
Table 1 Estimated concentrations of the detected pesticides

\begin{tabular}{llll}
\hline & $\begin{array}{l}\text { Robust Mean } \\
(\mathbf{m g} / \mathbf{k g})\end{array}$ & CV (\%) & $\begin{array}{l}\text { Estimated } \\
\text { concentration } \\
(\mathbf{m g} / \mathbf{k g})\end{array}$ \\
\hline Benalaxyl & 0.041 & 22 & 0.034 \\
Clomazone & 0.010 & 21 & 0.0085 \\
Emamectin & 0.0090 & 34 & 0.0090 \\
Etoxazole & 0.013 & 26 & 0.0098 \\
Fenpyrazamine & 0.0080 & 27 & 0.0085 \\
Isopryrazam & 0.0090 & 29 & 0.0012 \\
Metrafenone & 0.013 & 21 & 0.011 \\
Penflufen & 0.010 & 15 & 0.011 \\
Penthiopyrad & 0.067 & 23 & 0.067 \\
Prosulfocarb & 0.011 & 22 & 0.011 \\
Spinetoram & 0.035 & 26 & 0.029 \\
Spirotetramat & 0.072 & 38 & 0.012 \\
Sulfoxaflor & 0.0090 & 24 & 0.011 \\
Tetramethrin & 0.011 & 16 & 0.010 \\
\hline
\end{tabular}

by ChemSpider and EFS HRAM Compound database as proquinazid.

The standards of these analytes were available in our laboratory and the identified compounds were also quantified and the data processed. In Table 1, it is reported the estimated concentration of the identified pesticides. The detection limit ranged between 0.0025 to $0.0050 \mathrm{mg} / \mathrm{kg}$. Spirotetramat-enol was also present in the test item at the same range of concentration of others, but it was not detected; spirotetramat-ketohydroxy was present below $0.01 \mathrm{mg} / \mathrm{kg}$ and it was detected and reported and not all the laboratories reported the concentration.

\section{Case study 3}

For this test, it is available at this moment only a preliminary report by EURL, where it was reported which analytes were present in the test item, the list of laboratories that participated and the table with the compounds detected and non-detected by each laboratories. Only $17 \%$ of laboratories reported the entire results from all analytes, while $83 \%$ reported fewer analytes.

In this case study, no matrix reference was available as in a real routine case analysis.

The pesticide treatments were carried out post-harvest using standard analytical solutions. The spiked pesticides were 17: alachlor, cyanofenphos, diuron, dodemorph, endrin, fluacryprim, fonofos, isoprocarb, metamitron, metazachlor, metobromuron, monolinuron, prometryne, propazine, propoxur, simazine, and tetrachlorvinphos.

In this Proficiency Test, 64 laboratories participated and only three of them have not reported results. Thirteen pesticides $(76 \%$ of the reported pesticides by 
laboratory) were detected by our screening method using Compound Discoverer 2.0: diuron, dodemorph, fonofos, isoprocarb, metamitron, metazachlor, metobromuron, monolinuron, prometryne, propazine, propoxur, simazine, and tetrachlorvinphos (Supplementary Information, Additional file 2). Alachlor, cyanofenphos, endrin and fluacryprim were not reported.

Alachlor was not identified by Compound Discoverer 2.0 because it was not present its molecular ion. Our system identified its characteristic fragment $\left([\mathrm{M}+\mathrm{H}]^{+}=238.09875 \mathrm{~m} / \mathrm{z}\right)$ as ketamine $\left(\mathrm{C}_{13} \mathrm{H}_{16} \mathrm{ClNO}\right)$ at $8.072 \mathrm{~min}$ as retention time. Therefore, the putative identification of this analyte, that is not a stable compound, was compromised by the absence of the molecular ion and the fragment did not match to the library database. The database should be implemented with fragmentation pattern of the compound to better recognize a wide range of analytes.

The detection of cyanofenphos and fluacryprim was affected by an error of the laboratory operator. Onion is a very complex matrix, that presented a high number of compounds to be identified (about 395 compounds) and in this case it is necessary great attention in the elaboration and interpretation of the results and at last but not least, the adequately trained operator.

In a retrospective analysis, the cyanofenphos $\left(\mathrm{C}_{9} \mathrm{H}_{15} \mathrm{~N}_{5} \mathrm{OP}_{2} \mathrm{~S}\right)$ showed a low signal $[\mathrm{M}+\mathrm{H}]^{+}=304.05490 \mathrm{~m} / \mathrm{z}$ at retention time of $8.558 \mathrm{~min}$ with the presence also of its characteristic fragments $(156.98710$ and $258.01360 \mathrm{~m} / \mathrm{z})$. This signal was recognized by Chemspider database. In the case of fluacryprim $\left(\mathrm{C}_{20} \mathrm{H}_{21} \mathrm{~F}_{3} \mathrm{~N}_{2} \mathrm{O}_{5}\right)$, it was only detected by 20 laboratories. Its pseudomolecular ion $\left([\mathrm{M}+\mathrm{H}]^{+}=427.14670 \mathrm{~m} / \mathrm{z}\right)$ was present at retention time of $9.045 \mathrm{~min}$ and also the characteristic profile fragments in ddMS2 acquisition $(205.08582$ and $145.06476 \mathrm{~m} / \mathrm{z})$. This molecule was correctly identified by Compound Discoverer 2.0.

Finally, endrin is not an analyte suitable for the LC-MS determination and for this reason it was not detected.

These identifications were carried out without the analytical standards and the concentrations, for this reason, were not reported.

\section{Limitations}

This non targeted method presented in this paper presents some limitations.

For these kinds of analyses, it is really important the attention in the processing of data and the expertise of the operator. The data collected with this approach are many and need to be evaluated with the use of specific software to process data.
In the case of extremely complex matrices, like onion, it is possible, that the differential analysis cannot highlight signals of some analytes at low concentrations that could be covered by the matrix signals.

The databases contemplate only compounds that present parent ion and this is a limitation for the compounds instable that present a high fragmentation. The libraries could be implemented with the fragmentation pattern of the compound to help the identification.

\section{Conclusion}

The results of these studies are most encouraging and this method could be used more and more as screens/ filters, to make routine laboratory work easier and faster.

These three interlaboratory tests on wide-scope screening methods showed that such an approach can substantially expand the scope of xenobiotics analysis. This is especially useful for unknown compounds, like some pesticides, not frequently found in food and feed, or not monitored by the laboratories because they are not part of the EU-Coordinated Programme. The use of screening methods can greatly increase the chance of detecting less commonly found xenobiotics. However, the tests also revealed that verification of the screening methods performance (i.e. validation) are necessary to increase the reliability of such methods and guidelines for such validation have been prepared and included in the Document SANTE/12682/2019 (European Commission Directorate General for Health and Food Safety, 2019).

Furthermore, this screening method did not give false positive results.

In the near future, the perspectives should be focused on the implementation and development of database and software to help the identification of xenobiotics.

\section{Supplementary Information}

The online version contains supplementary material available at https://doi. org/10.1186/s40550-021-00086-x.

Additional file 1. Differential analysis and result table for sample material 1 (a) and sample material 2 (b) by means of Compound Discoverer 2.0.

Additional file 2. Identification results for the onion sample by means of Compound Discoverer 2.0 .

Acknowledgements

Not applicable.

Authors' contributions

All authors that contributed to the research paper are listed at the beginning. The author(s) read and approved the final manuscript. 


\section{Funding}

Not applicable.

\section{Availability of data and materials}

The RIKILT data are not accessible without a PT registration. It is possible to access the data with a specific request. Our lab code was 9837. For the EURL-FV10 (lab code 067), the data are available in https://www.eurlpesticides.eu/docs/public/tmplt_article.asp?CntID=1082\&LablD=500\&Lang= EN

Instead for EURL-FV12 (lab code 053), the data are available in https://www. eurl-pesticides.eu/userfiles/file//Preliminary_Report_SM12.pdf.

\section{Declarations}

\section{Competing interests}

Rossana Scarpone, Rachele Rocchi, Federico Bacà, Roberta Rosato, Francesco Chiumiento and Giacomo Migliorati declare that they have no conflict of interest.

\section{Received: 12 February 2021 Accepted: 18 October 2021}

Published online: 08 November 2021

\section{References}

Bletsou AA, Junho J, Hollender J, Archontaki E, Thomaidis NS (2015) Targeted and non-targeted liquid chromatography-mass spectrometric workflows for identification of transformation products of emerging pollutants in the aquatic environment. TrAC Trend Anal Chem 66:32-44. https://doi. org/10.1016/j.trac.2014.11.009

Cavanna D, Righetti L, Elliott C, Suman M (2018) The scientific challenges in moving from targeted to non-targeted mass spectrometric methods for food fraud analysis: a proposed validation workflow to bring about a harmonized approach. Trends Food Sci Technol 80:223-241. https://doi. org/10.1016/j.tifs.2018.08.007

Commission Decision n.657/EC (2002) Implementing Council Directive 96/23/ EC concerning the performance of analytical methods and the interpretation of results

EN 15662:2008 (2008) European Committee for Standardization (2008) CEN EN 15662: Foods of plant origin-determination of pesticide residues using GC-MS and/or LC-MS/MS following acetonitrile extraction/partition and clean-up by dispersive SPE-QuEChERS method. https://www.en-stand ard.eu/csn-en-15662-foods-of-plant-origin-multimethod-for-the-deter mination-of-pesticide-residues- using-gc-and-lc-based-analysis-follo wing-acetonitrile-extraction-partitioning-and-clean-up-by-dispersivespe-modular-quechers-method/

European Commission Directorate General for Health and Food Safety (2019) Analytical quality control and method validation for pesticide residues analysis in food and feed (SANTE/12682/2019), pp 1-48

Ferrer C, Lozano A, Agüera AA, Jiménez G, Fernández-Alba AR (2011) Overcoming matrix effects using the dilution approach in multiresidue methods for fruits and vegetables. J Chromatogr A 1218(42):7634-7639. https://doi.org/10.1016/j.chroma.2011.07.033

Furey A, Moriarty M, Bane V, Kinsella B, Lehane M (2013) Ion suppression; a critical review on causes, evaluation, prevention and applications. Talanta 115:104-122. https://doi.org/10.1016/j.talanta.2013.03.048

García-Reyes JF, Hernando MD, Molina-Díaz A, Fernández-Alba AR (2007) Comprehensive screening of target, non-target and unknown pesticides in food by LC-TOF-MS. TrAC Trend Anal Chem 26:828-841. https://doi. org/10.1016/j.trac.2007.06.006

Gosetti F, Mazzucco E, Zampieri D, Gennaro MC (2010) Signal suppression/ enhancement in high-performance liquid chromatography tandem mass spectrometry. J Chromatogr A 1217:3929-3937. https://doi.org/10.1016/j. chroma.2009.11.060

Guo C, Liping G, Weijian W, Jiawei L, Liming Z, Sheng X, Yanxia Z, Ruiqing X, Xunjie Z, Feng S (2020) Rapid screening and identification of targeted or non-targeted antitussive adulterants in herbal medicines by Q-Orbitrap HRAMS and screening database. Int J Mass Spectrom 447:116250. https:// doi.org/10.1016/j.jims.2019.116250

Herrera-Lopez S, Hernando MD, García-Calvo E, Fernández-Alba AR, Ulaszewska MM (2014) Simultaneous screening of targeted and nontargeted contaminants using an LC-QTOF-MS system and automated MS/MS library searching. J Mass Spectrom 49:878-893. https://doi.org/10.1002/ jms.3428

Hollender J, Van Bavel B, Dulio V, Farmen E, Furtmann K, Koschorreck J, Kunkel U, Krauss M, Munthe J, Schlabach M, Slobodnik J, Stroomberg G, Ternes T, Thomaidis NS, Togola A, Tornero V (2019) High resolution mass spectrometry-based non-target screening can support regulatory environmental monitoring and chemicals management. Environ Sci Eur 31(1):42-53. https://doi.org/10.1186/s12302-019-0225-x

Hu Q, Noll RJ, Li H, Makarov A, Hardmanc M, Cooks RG (2005) The Orbitrap: a new mass spectrometer. J Mass Spectrom 40:430-443. https://doi.org/10. 1002/jms.856

Kaufmann A (2014) Combining UHPLC and high-resolution MS: a viable approach for the analysis of complex samples? TrAC Trend Anal Chem 63:113-128. https://doi.org/10.1016/j.trac.2014.06.025

Kaufmann A, Butcher P, Maden K, Walker S, Widmer M (2015) Reliability of veterinary drug residue confirmation: high resolution mass spectrometry versus tandem mass spectrometry. Anal Chim Acta 856:54-67. https:// doi.org/10.1016/j.aca.2014.11.034

Ki NY, Jisu H, Beom KH, Kyung KH, Bong JM, Han BO, Jongki H (2019) Rapid screening of sulfonamides in dietary supplements based on extracted common ion chromatogram and neutral loss scan by LC-Q/TOF-mass spectrometry. J Food Drug Anal 27:164-174. https://doi.org/10.1016/j. jfda.2018.08.006

Makarov A, Scigelova M (2010) Coupling liquid chromatography to Orbitrap mass spectrometry. J Chromatogr A:3938-3945. https://doi.org/10.1016/j. chroma.2010.02.022

Milman BL (2015) General principles of identification by mass spectrometry. TrAC Trend Anal Chem 69:24-33. https://doi.org/10.1016/j.trac.2014.12. 009

Mol HGJ, Zomer P, De Koning M (2012) Qualitative aspects and validation of a screening method for pesticides in vegetables and fruits based on liquid chromatography coupled to full scan high resolution (Orbitrap) mass spectrometry. Anal Bioanal Chem 403:2891-2908. https://doi.org/10. 1007/s00216-012-6100-x

Peters RJB, Stolker AAM, Mol JGJ, Lommen A, Lyris E, Angelis Y, Vonaparti A, Stamou M, Georgakopoulos C, Nielen MWF (2010) Screening in veterinary drug analysis and sports doping control based on full-scan, accuratemass spectrometry. TrAC Trend Anal Chem 29:1250-1268. https://doi.org/ 10.1016/j.trac.2010.07.012

Riedl J, Esslinger S, Fauhl-Hassek C (2015) Review of validation and reporting of non-targeted fingerprinting approaches for food authentication. Anal Chim Acta 885:17-32. https://doi.org/10.1016/j.aca.2015.06.003

Scarpone R, Rosato R, Chiumiento F, Cipolletti C, Sergi M, Compagnone D (2020) Preliminary study to develop an alternative method for the nontargeted determination of xenobiotics in food by means of ultra high performance liquid chromatography coupled to high resolution and accuracy mass spectrometry. Food Anal Methods 13:1099-1110. https:// doi.org/10.1007/s12161-020-01727-1

Uclés S, Lozano A, Sosa A, Parrilla Vázquez P, Valverde A, Fernández-Alba AR (2017) Matrix interference evaluation employing GC and LC coupled to triple quadrupole tandem mass spectrometry. Talanta 174:72-81. https:// doi.org/10.1016/j.talanta.2017.05.068

US Pharmacopeia (2016) Guidance on developing and validating non-targeted methods for adulteration detection. Appendix XVIII:2053-2067

Zubarev RA, Makarov A (2013) Orbitrap mass spectrometry. Anal Chem 85:5288-5296. https://doi.org/10.1021/ac4001223

\section{Publisher's Note}

Springer Nature remains neutral with regard to jurisdictional claims in published maps and institutional affiliations. 